\title{
Stability of the Hartree equation with time-dependent coefficients
}

\author{
Binhua Feng ${ }^{1}$, Honghong Zhang ${ }^{1}$ and Yanjun Zhao ${ }^{2 *}$
}

"Correspondence: 940508086@qq.com

${ }^{2}$ College of Humanities and Sciences, Northeast Normal University, Changchun, 130117, P.R. China

Full list of author information is available at the end of the article

\section{Abstract}

In this paper, we investigate the stability for the nonlinear Hartree equation with time-dependent coefficients

$$
i \partial_{t} u+\Delta u+\alpha(t) \frac{1}{|x|} u+\beta(t)\left(W *|u|^{2}\right) u=0 .
$$

We first obtain the Lipschitz continuity of the solution $u=u(\alpha, \beta)$ with respect to coefficients $\alpha$ and $\beta$, and then prove that this equation is stable under the perturbation of coefficients. Our results improve some recent results.

MSC: 35Q55; 49J20

Keywords: nonlinear Hartree equation; stability; time-dependent coefficients; Lipschitz continuity

\section{Introduction}

Motivated by the nonlinearity management and dispersion management $[1,2]$ in the experimental work in Bose-Einstein condensates and optics, nonlinear Schrödinger equations have attracted more and more attention in both the physics and the mathematics fields; see [1-11] and the references therein.

In this paper, we consider the stability for the following nonlinear Schrödinger equation of Hartree type under the perturbation of coefficients:

$$
\left\{\begin{array}{l}
i \partial_{t} u+\Delta u+\alpha(t) \frac{1}{|x|} u+\beta(t)\left(W *|u|^{2}\right) u=0, \quad(t, x) \in[0, \infty) \times \mathbb{R}^{3}, \\
u(0, x)=u_{0}(x),
\end{array}\right.
$$

where $u(t, x)$ is a complex-valued function in $(t, x) \in[0, \infty) \times \mathbb{R}^{3}, u_{0} \in H^{1}\left(\mathbb{R}^{3}\right), \alpha(t)$ and $\beta(t)$ are two real-valued functions in $t \in[0, \infty)$.

Equation (1.1) arises as phenomenological models in many different contexts: HartreeFock theory, quantum field theory, etc. In particular, when $W=|x|^{-\gamma}$ with $0<\gamma<N$, this equation describes the mean-field limit of many-body quantum systems and has been extensively studied in $[4,12-22]$. An essential feature of the Hartree equations is that the convolution kernel $|x|^{-\gamma}$ still retains the fine structure of microscopic two-body interactions of the quantum system. Therefore, it is interesting to extend mathematical meth- 
ods developed for nonlinear Schrödinger equations with power nonlinearities $|u|^{p} u$ to the study of nonlinear Hartree equations.

When $\alpha(t)$ and $\beta(t)$ are two constants, Ma and Zhao in [23] investigated the stability of (1.1) under the perturbation of parameters. More precisely, they proved the following result.

Theorem A ([23], Theorem 1) Assume that $\alpha_{j}, \beta_{j}$ are positive numbers satisfying $\alpha_{j} \rightarrow 1$, $\beta_{j} \rightarrow 1$. Let $u_{j}$ be the $H^{1}$ solution of the following perturbed Hartree equation:

$$
i \partial_{t} u_{j}=\Delta u_{j}+\frac{\alpha_{j}}{|x|} u_{j}+\beta_{j}\left(\frac{1}{|x|} *\left|u_{j}\right|^{2}\right) u_{j} \quad \text { on } \mathbb{R}^{3},
$$

with initial data

$$
\left.u_{j}\right|_{t=0}=u(0) \in H^{1} .
$$

Then there exists a subsequence, still denoted by $u_{j}$, such that

$$
u_{j} \rightarrow u \quad \text { weakly in } L^{\infty}\left(H^{1}\right)
$$

where $u$ a weakly continuous solution of (1.1) with $\alpha(t) \equiv 1, \beta(t) \equiv 1$ and the initial data $u(0)$. Moreover, the energy and mass inequalities

$$
E(u(t)) \leq E(u(0)), \quad\|u(t)\|_{L^{2}} \leq\|u(0)\|_{L^{2}}, \quad \forall t>0,
$$

follow for this weak solution $u$, where

$$
E(u(t))=\int_{\mathbb{R}^{3}}\left[\frac{1}{2}|\nabla u(t, x)|^{2} d x-\frac{1}{4}\left(W *|u|^{2}\right)(x)|u(t, x)|^{2} d x-\frac{1}{2} \frac{|u(t, x)|^{2}}{|x|}\right] d x .
$$

In this paper, we will extend and improve this result in several aspects:

1. Our results hold for more general Hartree nonlinearities $\left(W *|u|^{2}\right) u$, where $W \in L^{p}\left(\mathbb{R}^{3}\right)+L^{\infty}\left(\mathbb{R}^{3}\right)$ for some $p \geq 1$.

2. We extend this result to the time-dependent coefficients $\alpha(t)$ and $\beta(t)$.

3. We prove the locally Lipschitz continuity of the solution $u(\alpha, \beta)$ with respect to the coefficients $\alpha$ and $\beta$.

4. We prove that $u_{j}$ strongly converge to $u$ in $L^{\gamma}\left((0, T), W^{1, \rho}\right)$ as $j \rightarrow \infty$, for every admissible pair $(\gamma, \rho)$ and all $0<T<T^{*}$.

More precisely, we will prove the following results.

Theorem 1.1 Let $W: \mathbb{R}^{3} \rightarrow \mathbb{R}$ be an even, real-valued function and $W \in L^{p}\left(\mathbb{R}^{3}\right)+L^{\infty}\left(\mathbb{R}^{3}\right)$ for some $p \geq 1, \alpha_{j} \rightarrow \alpha$ and $\beta_{j} \rightarrow \beta$ in $H^{1}\left(0, T^{*}\right)$. Assume that $u$ is the solution of (1.1) defined on the maximal interval $\left[0, T^{*}\right)$ with the initial value $u_{0} \in H^{1}$. Suppose that $u_{j}$ is the solution to the equation

$$
\left\{\begin{array}{l}
i \partial_{t} u+\Delta u+\alpha_{j}(t) \frac{1}{|x|} u+\beta_{j}(t)\left(W *|u|^{2}\right) u=0, \\
u(0, x)=u_{0}(x) .
\end{array}\right.
$$


Then:

(i) Given any $0<T<T^{*}$, the solution $u_{j}$ exists on $[0, T]$ if $j$ is sufficiently large.

(ii) For every admissible pair $(\gamma, \rho)$ and $0<T<T^{*}, u_{j} \rightarrow u$ in $L^{\gamma}\left((0, T), W^{1, \rho}\right)$ as $j \rightarrow \infty$. In particular, convergence holds in $C\left([0, T], H^{1}\right)$ for $0<T<T^{*}$. In addition, for every admissible pair $(\gamma, \rho)$

$$
\left\|u_{j}-u\right\|_{L^{\gamma}\left((0, T), W^{1, \rho}\right)} \leq C\left\|\alpha_{j}-\alpha\right\|_{H^{1}(0, T)}+C\left\|\beta_{j}-\beta\right\|_{H^{1}(0, T)},
$$

where $C$ depends on $u_{0}, T, \gamma, \rho$.

Remark For physical reasons, in this paper, we only study the three-dimensional case. In fact, we can investigate more general unbounded potentials in $\mathbb{R}^{N}$. Our results also follow if the potential $V: \mathbb{R}^{N} \rightarrow \mathbb{R}$ is a real-valued function, satisfying:

(V1) $V \in L^{q}\left(\mathbb{R}^{N}\right)+L^{\infty}\left(\mathbb{R}^{N}\right)$ for some $q \geq 1, q>N / 2$;

(V2) $V, \nabla V \in L^{q}\left(\mathbb{R}^{N}\right)+L^{\infty}\left(\mathbb{R}^{N}\right)$ for some $q \geq 2, q>N / 2$.

A typical example satisfying these assumptions on $V$ is $\frac{1}{|x|^{\alpha}}$ for some $\alpha>0$.

This paper is organized as follows: in Section 2, we will collect some preliminaries such as local well-posedness, global existence and a Gronwall-type estimate. In Section 3, we firstly obtain the Lipschitz continuity of the solution $u=u(\alpha, \beta)$ with respect to coefficients $\alpha$ and $\beta$, and then prove Theorem 1.1.

Notation In this paper, we use the following notation. $C>0$ denotes various positive constants. Because we only consider $\mathbb{R}^{3}$, we often use the abbreviations $L^{r}=L^{r}\left(\mathbb{R}^{3}\right)$, $H^{1}=H^{1}\left(\mathbb{R}^{3}\right)$. Given any interval $I \subset \mathbb{R}$, the norms of mixed spaces $L^{q}\left(I, L^{r}\left(\mathbb{R}^{3}\right)\right)$ and $L^{q}\left(I, H^{s}\left(\mathbb{R}^{3}\right)\right)$ are denoted by $\|\cdot\|_{L_{t}^{q} L_{x}^{r}(I)}$ and $\|\cdot\|_{L^{q}\left(I, H^{s}\right)}$, respectively. We recall that a pair $(q, r)$ is admissible if $\frac{2}{q}=3\left(\frac{1}{2}-\frac{1}{r}\right)$ and $2 \leq r \leq 6$. For simplicity, we always denote $V_{1}=\frac{1}{|x|} \chi_{B_{0}}$ and $V_{2}=\frac{1}{|x|}\left(1-\chi_{B_{0}}\right)$, where $B_{0}$ is the unit ball in $\mathbb{R}^{3}$ centered at the original point, $\chi_{B_{0}}$ is its characteristic function. It is obvious that $V_{1} \in L^{\frac{3}{1+3 \varepsilon}}, \nabla V_{1} \in L^{\frac{3}{2+3 \varepsilon}}$ with $\varepsilon>0$ sufficiently small, $V_{2} \in L^{\infty}$ and $\nabla V_{2} \in L^{\infty}$.

\section{Preliminaries}

Firstly, we investigate the local well-posedness for (1.1). When $\alpha$ and $\beta$ are two constants, the local well-posedness of (1.1) has been studied in [4]. In our case, when the terms $\alpha \frac{u}{|x|}$ and $\beta W *|u|^{2} u$ have to be estimated in some norms, due to $\alpha, \beta \in H^{1}(0, \infty) \hookrightarrow L^{\infty}(0, \infty)$, we only need to take $L^{\infty}$ norms of $\alpha$ and $\beta$. Therefore, by a similar method as that in [4], we can prove the local well-posedness of (1.1).

Lemma 2.1 Assume that $W: \mathbb{R}^{3} \rightarrow \mathbb{R}$ is an even, real-valued potential and $W \in L^{p}+$ $L^{\infty}$ for some $p \geq 1$. Given $A, B, M>0$, there exists $\delta=\delta(A, B, M)$ such that, for all $\alpha, \beta \in$ $H^{1}(0, \infty)$ satisfying $\|\alpha\|_{L^{\infty}} \leq A,\|\beta\|_{L^{\infty}} \leq B$ and all $u_{0} \in H^{1}$ satisfying $\left\|u_{0}\right\|_{H^{1}} \leq M$, there exists a unique solution $u \in C\left([0, \delta], H^{1}\right)$ of $(1.1)$ and $\|u\|_{L^{\infty}\left((0, \delta), H^{1}\right)} \leq 2\left\|u_{0}\right\|_{H^{1}}$. In addition, the solution $u$ of (1.1) satisfies

$$
\|u(t)\|_{L^{2}}=\left\|u_{0}\right\|_{L^{2}}
$$


and

$$
\frac{d}{d t} E(t)=-\frac{\alpha^{\prime}(t)}{2} \int_{\mathbb{R}^{3}} \frac{|u(t, x)|^{2}}{|x|} d x-\frac{\beta^{\prime}(t)}{4} \int_{\mathbb{R}^{3}}\left(W *|u|^{2}\right)(x)|u(t, x)|^{2} d x
$$

for all $t \in[0, \delta]$, where

$$
E(u(t))=\int_{\mathbb{R}^{3}}\left[\frac{1}{2}|\nabla u(t, x)|^{2}-\frac{\alpha(t)}{2} \frac{|u(t, x)|^{2}}{|x|}-\frac{\beta(t)}{4}\left(W *|u|^{2}\right)(x)|u(t, x)|^{2}\right] d x .
$$

Next, we consider the global existence for (1.1).

Lemma 2.2 Let $W: \mathbb{R}^{3} \rightarrow \mathbb{R}$ be an even, real-valued potential and $W \in L^{\sigma}+L^{\infty}$ for some $\sigma>3 / 2$. If $u_{0} \in H^{1}$, then the solution $u(t)$ of (1.1) exists globally.

Proof We first deduce from Hölder's and Young's inequalities that

$$
\int_{\mathbb{R}^{3}} \frac{|u(x)|^{2}}{|x|} d x \leq C\left\|V_{1}\right\|_{L^{1+3 \varepsilon}} \frac{3}{\|}\|\|_{L^{\frac{6}{2-3 \varepsilon}}}^{2}+C\left\|V_{2}\right\|_{L^{\infty}}\|u\|_{L^{2}}^{2}
$$

and

$$
\int_{\mathbb{R}^{3}}\left(W *|u|^{2}\right)|u|^{2} d x \leq C\left\|W_{1}\right\|_{L^{\sigma}}\|u\|_{L^{\frac{4 \sigma}{2 \sigma-1}}}^{4}+C\left\|W_{2}\right\|_{L^{\infty}}\|u\|_{L^{2}}^{4}
$$

where $W=W_{1}+W_{2}$ with $W_{1} \in L^{\sigma}$ and $W_{2} \in L^{\infty}$.

Thus, by (2.2), (2.4) and (2.5), we derive

$$
\begin{aligned}
\left\|E^{\prime}\right\|_{L^{2}(0, T)} \leq & C\left\|\alpha^{\prime}\right\|_{L^{2}(0, T)}\left(\left\|V_{1}\right\|_{L^{\frac{3}{1+3 \varepsilon}}}\|u\|_{L^{2-3 \varepsilon}}^{2}+\left\|V_{2}\right\|_{L^{\infty}}\left\|u_{0}\right\|_{L^{2}}^{2}\right) \\
& +C\left\|\beta^{\prime}\right\|_{L^{2}(0, T)}\left(\left\|W_{1}\right\|_{L^{\sigma}}\|u\|_{L^{\frac{4 \sigma}{2 \sigma-1}}}^{4}+\left\|W_{2}\right\|_{L^{\infty}}\left\|u_{0}\right\|_{L^{2}}^{4}\right) .
\end{aligned}
$$

This and (2.3) yield

$$
\begin{aligned}
E(t)= & E(0)+\int_{0}^{t} E^{\prime}(s) d s \leq E(0)+\left(t \int_{0}^{t}\left(E^{\prime}(s)\right)^{2} d s\right)^{1 / 2} \\
\leq & E(0)+C t^{1 / 2}\left\|\alpha^{\prime}\right\|_{L^{2}(0, T)}\left(\left\|V_{1}\right\|_{L^{1+3 \varepsilon}}\|u\|_{L^{2-3 \varepsilon}}^{2}+\left\|V_{2}\right\|_{L^{\infty}}\left\|u_{0}\right\|_{L^{2}}^{2}\right) \\
& +C t^{1 / 2}\left\|\beta^{\prime}\right\|_{L^{2}(0, T)}\left(\left\|W_{1}\right\|_{L^{\sigma}}\|u\|_{L_{\frac{4 \sigma}{2 \sigma-1}}}^{4}+\left\|W_{2}\right\|_{L^{\infty}}\left\|u_{0}\right\|_{L^{2}}^{4}\right) .
\end{aligned}
$$

Combining (2.2), (2.4)-(2.6), we obtain

$$
\begin{aligned}
\int_{\mathbb{R}^{3}}|\nabla u(t, x)|^{2} d x \leq & E(u(t))+C \int_{\mathbb{R}^{3}} \frac{|u(t, x)|^{2}}{|x|} d x+C \int_{\mathbb{R}^{3}}\left(W *|u|^{2}\right)(x)|u(t, x)|^{2} d x \\
\leq & E(0)+C\left\|V_{1}\right\|_{L^{1+3 \varepsilon}}\|u\|_{L^{\frac{6}{2-3 \varepsilon}}}^{2}+C\left\|V_{2}\right\|_{L^{\infty}}\left\|u_{0}\right\|_{L^{2}}^{2} \\
& +C\left\|W_{1}\right\|_{L^{\sigma}}\|u\|_{L^{\frac{4 \sigma}{2 \sigma-1}}}^{4}+C\left\|W_{2}\right\|_{L^{\infty}}\left\|u_{0}\right\|_{L^{2}}^{4} .
\end{aligned}
$$

On the other hand, we have the following Gagliardo-Nirenberg's inequalities:

$$
\|u\|_{L^{2}-3 \varepsilon}^{2} \leq C\|u\|_{H^{1}}^{1+3 \varepsilon}\|u\|_{L^{2}}^{1-3 \varepsilon}
$$




$$
\|u\|_{L^{\frac{4 \sigma}{2 \sigma-1}}}^{4} \leq C\|u\|_{H^{1}}^{\frac{3}{\sigma}}\|u\|_{L^{2}}^{\frac{4 \sigma-3}{\sigma}}
$$

Taking $\varepsilon$ such that $1+3 \varepsilon<2$, and $\frac{3}{\sigma}<2$, we infer from (2.7) and Young's inequality with $\varepsilon$ that

$$
\|u(t)\|_{H^{1}} \leq C\left(T,\left\|u_{0}\right\|_{H^{1}},\|\alpha\|_{H^{1}(0, T)},\|\beta\|_{H^{1}(0, T)}\right) \quad \text { for every } t \in[0, T]
$$

for every $0<T<\infty$. This implies that the solution $u$ of (1.1) is global.

Finally, we recall a Gronwall-type estimate which is vital to obtain the Lipschitz continuity of solution $u(\alpha, \beta)$ with respect to coefficients $\alpha$ and $\beta$.

Lemma 2.3 ([24]) Let $T>0,1 \leq p_{2}<q_{2} \leq \infty, 1 \leq p_{1}<q_{1} \leq \infty$, and $a, b_{1}, b_{2} \geq 0$. If $f_{1} \in$ $L^{q_{1}}(0, T), f_{2} \in L^{q_{2}}(0, T)$ satisfying

$$
\left\|f_{1}\right\|_{L^{q_{1}(0, t)}}+\left\|f_{2}\right\|_{L^{q_{2}(0, t)}} \leq a+b_{1}\left\|f_{1}\right\|_{L^{p_{1}(0, t)}}+b_{2}\left\|f_{2}\right\|_{L^{p_{2}(0, t)}},
$$

for all $0<t<T$, then there exists $\Gamma=\Gamma\left(b_{1}, b_{2}, p, q, T\right)$ such that

$$
\left\|f_{1}\right\|_{L^{q_{1}(0, T)}}+\left\|f_{2}\right\|_{L^{q_{2}(0, T)}} \leq a \Gamma \text {. }
$$

\section{The proof of main results}

In this section, we first prove that the solution of (1.1) depends local Lipschitz continuously on the coefficients $\alpha$ and $\beta$, and then show our main results.

Lemma 3.1 Assume that $W: \mathbb{R}^{3} \rightarrow \mathbb{R}$ is an even, real-valued function and $W \in L^{p}+L^{\infty}$ for some $p \geq 1$. Given $u_{0} \in H^{1}$. Let $u \in L^{\infty}\left(\left[0, T^{*}\right), H^{1}\right)$ be the corresponding solution of (1.1) with coefficients $\alpha, \beta \in H^{1}(0, T)$. There exists $\varepsilon>0$ such that if $\tilde{\alpha}$ and $\tilde{\beta}$ satisfy $\| \tilde{\alpha}-$ $\alpha\left\|_{H^{1}(0, T)}<\varepsilon,\right\| \tilde{\beta}-\beta \|_{H^{1}(0, T)}<\varepsilon$ and $\tilde{u}$ is the corresponding solution of (1.1) with coefficients $\tilde{\alpha}$ and $\tilde{\beta}$. Then:

(i) Given any $0<T<T^{*}$, the solution $\tilde{u}$ exists on $[0, T]$.

(ii) For every admissible pair $(\gamma, \rho)$

$$
\|\tilde{u}-u\|_{L^{\gamma}\left((0, T), W^{1, \rho}\right)} \leq C\|\tilde{\alpha}-\alpha\|_{H^{1}(0, T)}+C\|\tilde{\beta}-\beta\|_{H^{1}(0, T)}
$$

where $C$ depends on $u_{0}, T, \gamma, \rho$. In particular,

$$
\|\tilde{u}-u\|_{L^{\infty}\left((0, T), H^{1}\right)} \leq C\|\tilde{\alpha}-\alpha\|_{H^{1}(0, T)}+C\|\tilde{\beta}-\beta\|_{H^{1}(0, T)}
$$

Proof Firstly, we assume that the solution $\tilde{u}$ exists on $[0, T]$. Note that the following Duhamel's formulation:

$$
u(t)=U(t) u_{0}+i \int_{0}^{t} U(t-s)\left(\alpha(s) \frac{1}{|x|} u(s)+\left(\beta(s) W *|u(s)|^{2}\right) u(s)\right) d s
$$

where $U(t):=e^{i t \Delta}$ denotes the free Schrödinger propagator, which is isometric on $H^{s}$ for every $s \geq 0$; see [4]. This yields

$$
\tilde{u}(t)-u(t)=i \int_{0}^{t} U(t-r)\left(\frac{1}{|x|}(\tilde{\alpha} \tilde{u}-\alpha u)+\left(\tilde{\beta}\left(W *|\tilde{u}|^{2}\right) \tilde{u}-\beta\left(W *|u|^{2}\right) u\right)\right)(r) d r .
$$


In the following, we set $\rho_{1}=\frac{4 p}{2 p-1}$, taking $\gamma_{1}$ such that $\left(\gamma_{1}, \rho_{1}\right)$ is an admissible pair. Applying Strichartz's estimate to (3.3), we deduce from Hölder's inequality that for $0<t \leq T$

$$
\begin{aligned}
& \|\tilde{u}-u\|_{L_{t}^{\gamma} L_{x}^{\rho}(0, t)} \\
& \leq C\left\|\tilde{\beta}\left(W *|\tilde{u}|^{2}\right) \tilde{u}-\tilde{\beta}\left(W *|u|^{2}\right) u\right\|_{L_{t}^{\gamma_{1}^{\prime}} L_{x}^{\rho_{1}^{\prime}}(0, t)} \\
& +C\left\|(\tilde{\beta}-\beta)\left(W *|u|^{2}\right) u\right\|_{L_{t}^{\gamma_{1}^{\prime}} L_{x}^{\rho_{1}^{\prime}}(0, t)} \\
& +C\left\|V_{1}(\tilde{\alpha} \tilde{u}-\alpha u)\right\|_{L_{t}^{2} L_{x}^{\frac{6}{5}}(0, t)}+C\left\|V_{2}(\tilde{\alpha} \tilde{u}-\alpha u)\right\|_{L_{t}^{1} L_{x}^{2}(0, t)} \\
& \leq C\|\tilde{\beta}\|_{L^{\infty}(0, t)}\left(\|\tilde{u}\|_{L_{t}^{\infty} L_{x}^{\rho_{1}(0, t)}}^{2}+\|u\|_{L_{t}^{\infty} L_{x}^{\rho_{1}(0, t)}}^{2}\right)\|\tilde{u}-u\|_{L_{t}^{\gamma_{1}^{\prime}} L_{x}^{\rho_{1}}(0, t)} \\
& +C\|\tilde{\beta}-\beta\|_{L^{\infty}(0, t)}\|\tilde{u}\|_{L_{t}^{\infty} L_{x}^{\rho_{1}}(0, t)}^{3}+C\left\|V_{1}\right\|_{L^{1+3 \varepsilon}}\|\tilde{\alpha}\|_{L^{\infty}(0, t)}\|\tilde{u}-u\|_{L_{t}^{2} \frac{2}{L_{x}^{1-2 \varepsilon}}(0, t)} \\
& +\left\|V_{1}\right\|_{L^{1+3 \varepsilon}}\|\tilde{\alpha}-\alpha\|_{L^{\infty}(0, t)}\|u\|_{L_{t}^{2} L_{x}^{1-2 \varepsilon}} \frac{2}{10, t)} \\
& +C\left\|V_{2}\right\|_{L^{\infty}}\|\tilde{\alpha}\|_{L^{\infty}(0, t)}\|\tilde{u}-u\|_{L_{t}^{1} L_{x}^{2}(0, t)}+\left\|V_{2}\right\|_{L^{\infty}}\|\tilde{\alpha}-\alpha\|_{L^{\infty}(0, t)}\|u\|_{L_{t}^{1} L_{x}^{2}(0, t)} \\
& \leq C\|\tilde{u}-u\|_{L_{t}^{\gamma_{1}^{\prime}} L_{x}^{\rho_{1}}(0, t)}+C\|\tilde{u}-u\|_{L_{t}^{2} L_{x}^{1-2 \varepsilon}(0, t)}+C\|\tilde{u}-u\|_{L_{t}^{1} L_{x}^{2}(0, t)} \\
& +C\|\tilde{\alpha}-\alpha\|_{H^{1}(0, t)}+C\|\tilde{\beta}-\beta\|_{H^{1}(0, t)},
\end{aligned}
$$

which, together with Lemma 2.3, implies that for every admissible pair $(\gamma, \rho)$

$$
\|\tilde{u}-u\|_{L_{t}^{\gamma} L_{x}^{\rho}(0, T)} \leq C\|\tilde{\alpha}-\alpha\|_{H^{1}(0, T)}+C\|\tilde{\beta}-\beta\|_{H^{1}(0, T)},
$$

where $C$ depends on $\|\tilde{u}\|_{L^{\infty}\left((0, T), H^{1}\right)},\|u\|_{L^{\infty}\left((0, T), H^{1}\right)}, T, \gamma$. In addition, by a similar argument to that of (3.4), the embedding $W^{1, \frac{2}{1-2 \varepsilon}} \hookrightarrow L^{\frac{6}{1-6 \varepsilon}}$, we obtain

$$
\begin{aligned}
\| \nabla \tilde{u}- & \nabla u \|_{L_{t}^{\gamma} L_{x}^{\rho}(0, t)} \\
\leq & C\left\|\tilde{\beta}\left(W *|\tilde{u}|^{2}\right) \nabla \tilde{u}-\beta\left(W *|u|^{2}\right) \nabla u\right\|_{L_{t}^{\gamma_{1}^{\prime}} L_{x}^{\rho_{1}^{\prime}}(0, t)} \\
& +C\left\|\tilde{\beta}\left(W * \nabla|\tilde{u}|^{2}\right) \tilde{u}-\beta\left(W * \nabla|u|^{2}\right) u\right\|_{L_{t}^{\gamma_{1}^{\prime}} L_{x}^{\rho_{1}^{\prime}}(0, t)} \\
& +C\left\|(\tilde{\alpha}-\alpha) \nabla V_{1} \tilde{u}\right\|_{L_{t}^{2} L_{x}^{\frac{6}{5}}(0, t)}+C\left\|\alpha \nabla V_{1}(\tilde{u}-u)\right\|_{L_{t}^{2} L_{x}^{5}(0, t)} \\
& +C\left\|(\tilde{\alpha}-\alpha) \nabla V_{2} \tilde{u}\right\|_{L_{t}^{1} L_{x}^{2}(0, t)}+C\left\|\alpha \nabla V_{2}(\tilde{u}-u)\right\|_{L_{t}^{1} L_{x}^{2}(0, t)} \\
& +C\left\|(\tilde{\alpha}-\alpha) V_{1} \nabla \tilde{u}\right\|_{L_{t}^{2} L_{x}^{\frac{6}{5}}(0, t)}+C\left\|\alpha V_{1} \nabla(\tilde{u}-u)\right\|_{L_{t}^{2} L_{x}^{\frac{6}{5}}(0, t)} \\
& +C\left\|(\tilde{\alpha}-\alpha) V_{2} \nabla \tilde{u}\right\|_{L_{t}^{1} L_{x}^{2}(0, t)}+C\left\|\alpha V_{2} \nabla(\tilde{u}-u)\right\|_{L_{t}^{1} L_{x}^{2}(0, t)} \\
\leq & C\|\tilde{u}-u\|_{L_{t}^{\gamma_{1}} L_{x}^{\rho_{1}}(0, t)}+C\|\nabla(\tilde{u}-u)\|_{L_{t}^{\gamma_{1}^{\prime}} L_{x}^{\rho_{1}^{1}}(0, t)} \\
& +C\|\tilde{\alpha}-\alpha\|_{H^{1}(0, t)}+C\|\tilde{\beta}-\beta\|_{H^{1}(0, t)}+C\|\tilde{u}-u\|_{L_{t}^{1} L_{x}^{2}(0, t)} \\
& +C\|\nabla(\tilde{u}-u)\|_{L_{t}^{2} L_{x}^{1-2 \varepsilon}(0, t)}+C\|\nabla(\tilde{u}-u)\|_{L_{t}^{1} L_{x}^{2}(0, t)} .
\end{aligned}
$$


Hence, it follows from Lemma 2.3 and (3.5) that for every admissible pair $(\gamma, \rho)$

$$
\|\tilde{u}-u\|_{L^{\gamma}\left((0, T), W^{1, \rho}\right)} \leq C\|\tilde{\alpha}-\alpha\|_{H^{1}(0, T)}+C\|\tilde{\beta}-\beta\|_{H^{1}(0, T)},
$$

where $C$ depends on $\left\|u_{j}\right\|_{L^{\infty}\left((0, T), H^{1}\right)},\|u\|_{L^{\infty}\left((0, T), H^{1}\right)}, T, \gamma$.

Therefore, in order to prove this lemma, we only need to show that there exist $\varepsilon>0$ and $M>0$ such that if $\tilde{\alpha}$ and $\tilde{\beta}$ satisfy $\|\tilde{\alpha}-\alpha\|_{H^{1}(0, T)}<\varepsilon$ and $\|\tilde{\beta}-\beta\|_{H^{1}(0, T)}<\varepsilon$, then the corresponding solution $\tilde{u}$ exists on $[0, T]$ and $\|\tilde{u}\|_{L^{\infty}\left((0, T), H^{1}\right)} \leq M$.

To this aim, we set

$$
M=2 \sup _{0 \leq t \leq T}\|u(t)\|_{H^{1}}+1
$$

With the notation of Lemma 2.1, let $\delta=\delta(A, B, M)$, where $A=\|\tilde{\alpha}\|_{L^{\infty}}, B=\|\tilde{\beta}\|_{L^{\infty}}$ and $M$ is given by (3.8). We infer from Lemma 2.1 that $\tilde{u}$ exists on $[0, \delta]$ and that

$$
\|\tilde{u}\|_{L^{\infty}\left((0, \delta), H^{1}\right)} \leq 2\left\|u_{0}\right\|_{H^{1}}
$$

On the other hand, by (3.7), we have

$$
\|\tilde{u}(\delta)-u(\delta)\|_{H^{1}} \leq C\|\tilde{\alpha}-\alpha\|_{H^{1}(0, T)}+C\|\tilde{\beta}-\beta\|_{H^{1}(0, T)} .
$$

Taking $\varepsilon$ such that $C \varepsilon<M / 4$, it follows that $\|\tilde{u}(\delta)\|_{H^{1}}<M$. Hence, we can repeat the argument to continue the solution also in the time interval $[\delta, 2 \delta]$, and so on. Since the solution $u(t)$ exists on $\left[0, T^{*}\right)$, and for any $0<T<T^{*}$, we consider $[0, T] \subset[0, \delta] \cup \cdots \cup[(N-$ 1) $\delta, N \delta], N=\left[\frac{T}{\delta}\right]+1$, where $[\cdot]$ denotes the integer part of the number. Thus, the solution $\tilde{u}$ exists on $[0, T]$ and $\|\tilde{u}\|_{L^{\infty}\left((0, T), H^{1}\right)} \leq M$. This completes the proof.

Proof of Theorem 1.1 Given $T \in\left(0, T^{*}\right)$. Let $A=\|\alpha\|_{L^{\infty}(0, T)}, B=\|\beta\|_{L^{\infty}(0, T)}$ and $M=$ $2 \sup _{0 \leq t \leq T}\|u(t)\|_{H^{1}}+1$. With the notation of Lemma 2.1, setting $\delta=\delta(A, B, M)$. We infer from Lemma 2.1 that $u_{j}$ exists on $[0, \delta]$ and satisfies

$$
\limsup _{j \rightarrow \infty}\left\|u_{j}\right\|_{L^{\infty}\left((0, \delta), H^{1}\right)}<2\left\|u_{0}\right\|_{H^{1}}
$$

Applying Lemma 3.1, we see that the conclusion holds on the interval $[0, \delta]$.

Let $0<l \leq T$ be such that $u_{j}$ exists on $[0, l]$ for $j$ sufficiently large and

$$
\limsup _{j \rightarrow \infty}\left\|u_{j}\right\|_{L^{\infty}\left((0, l), H^{1}\right)}<\infty
$$

Then we infer from Lemma 3.1 that

$$
u_{j} \rightarrow u \quad \text { in } L^{\gamma}\left((0, l), W^{1, \rho}\right) \text { as } j \rightarrow \infty
$$

for every admissible pair $(\gamma, \rho)$. In particular, $u_{j}(l) \rightarrow u(l)$ in $H^{1}$ as $j \rightarrow \infty$, which, together with the definition of $M$ yields that $\left\|u_{j}(l)\right\|_{H^{1}}<M$. Applying Lemma 2.1 with the initial value $u_{j}(l)$, it follows that $u_{j}$ exists on $[0, l+\delta]$ and

$$
\limsup _{j \rightarrow \infty}\left\|u_{j}\right\|_{L^{\infty}\left((0, l+\delta), H^{1}\right)} \leq C
$$


It follows from Lemma 3.1 that the estimate (3.13) holds with $l$ replaced by $l+\delta$ provided $l+\delta \leq T$. Iterating this argument, we see that

$$
\limsup _{j \rightarrow \infty}\left\|u_{j}\right\|_{L^{\infty}\left((0, T), H^{1}\right)} \leq C
$$

This estimate with Lemma 3.1 yields the desired results.

\section{Conclusions}

In this paper, we consider the stability for the nonlinear Schrödinger equation (1.1) of Hartree type under the perturbation of coefficients. We first obtain the Lipschitz continuity of the solution $u=u(\alpha, \beta)$ with respect to coefficients $\alpha$ and $\beta$ by using Strichartz's estimates, and then prove that this equation is stable under the perturbation of coefficients by a bootstrap argument. Our results improve some recent results. In particular, the proof of the locally Lipschitz continuity contains a very general method that may be useful for other related problems.

Funding

This work is supported by Gansu Provincial Natural Science Foundation (1606RJZA010) and NWNU-LKQN-14-6.

Competing interests

The authors declare that no competing interests exist.

Authors' contributions

The authors contributed equally to this paper. All authors read and approved the final manuscript.

\section{Author details}

${ }^{1}$ Department of Mathematics, Northwest Normal University, Lanzhou, 730070, P.R. China. ${ }^{2}$ College of Humanities and Sciences, Northeast Normal University, Changchun, 130117, P.R. China.

\section{Publisher's Note}

Springer Nature remains neutral with regard to jurisdictional claims in published maps and institutional affiliations.

Received: 2 March 2017 Accepted: 9 August 2017 Published online: 31 August 2017

\section{References}

1. Malomed, BA: Soliton Management in Periodic Systems. Springer, Berlin (2006)

2. Centurion, M, Porter, MA, Kevrekidis, PG, Psaltis, D: Nonlinearity management in optics: experiment, theory, and simulation. Phys. Rev. Lett. 97, 033903 (2006)

3. Antonelli, P, Saut, J, Sparber, C: Well-posedness and averaging of NLS with time-periodic dispersion management. Adv. Differ. Equ. 18, 1/2 (2013)

4. Cazenave, T: Semilinear Schrödinger Equations. Courant Lecture Notes in Mathematics, vol. 10. Am. Math. Soc., Providence (2003)

5. Bhattarai, S: Stability of solitary-wave solutions of coupled NLS equations with power-type nonlinearities. Adv. Nonlinear Anal. 4, 73-90 (2015)

6. Ghergu, M, Radulescu, V: Nonlinear PDEs. Mathematical Models in Biology, Chemistry and Population Genetics. Springer Monographs in Mathematics. Springer, Heidelberg (2012)

7. Bisci, GM, Radulescu, V: Ground state solutions of scalar field fractional Schrödinger equations. Calc. Var. Partial Differ. Equ. 54, 2985-3008 (2015)

8. Cazenave, T, Scialom, M: A Schrödinger equation with time-oscillating nonlinearity. Rev. Mat. Univ. Complut. Madr. $23,321-339(2010)$

9. Feng, B, Zhao, D, Sun, C: The limit behavior of solutions for the nonlinear Schrödinger equation including nonlinear loss/gain with variable coefficient. J. Math. Anal. Appl. 405, 240-251 (2013)

10. Feng, B, Zhao, D, Sun, C: On the Cauchy problem for the nonlinear Schrödinger equations with time-dependent linear loss/gain. J. Math. Anal. Appl. 416, 901-923 (2014)

11. Feng, B, Zhao, D, Sun, C: Homogenization for nonlinear Schrödinger equations with periodic nonlinearity and dissipation in fractional order spaces. Acta Math. Sci. Ser. B Engl. Ed. 35, 567-582 (2015)

12. Ablowitz, MJ, Prinari, B, Trubatch, AD: Discrete and Continuous Nonlinear Schrödinger Systems. Cambridge University Press, Cambridge (2004)

13. Marin, M: A temporally evolutionary equation in elasticity of micropolar bodies with voids. Bull. Ser. Appl. Math. Phys. 60, 3-12 (1998) 
14. Marin, M, Baleanu, D: On vibrations in thermoelasticity without energy dissipation for micropolar bodies. Bound. Value Probl. 2016, 1 (2016)

15. Marin, M, Marinescu, C: Thermoelasticity of initially stressed bodies, asymptotic equipartition of energies. Int. J. Eng. Sci. 36, 73-86 (1998)

16. Marin, M, Agarwal, RP, Codarcea, L: A mathematical model for three-phase-lag dipolar thermoelastic bodies. Arch. Inequal. Appl. 2017, 109 (2017)

17. Sulem, C, Sulem, PL: The Nonlinear Schrödinger Equation. Springer, Berlin (1999)

18. Feng, B, Yuan, X: On the Cauchy problem for the Schrödinger-Hartree equation. Evol. Equ. Control Theory 4, 431-445 (2015)

19. Feng, B, Wang, K: Optimal bilinear control of nonlinear Hartree equations with singular potentials. J. Optim. Theory Appl. 170, 756-771 (2016)

20. Feng, B, Liu, J, Zheng, J: Optimal bilinear control of nonlinear Hartree equation in $\mathbb{R}^{3}$. Electron. J. Differ. Equ. 2013, 130 (2013)

21. Feng, B: Sharp threshold of global existence and instability of standing wave for the Schrödinger-Hartree equation with a harmonic potential. Nonlinear Anal., Real World Appl. 31, 132-145 (2016)

22. Miao, $C, X u, G, Z$ hao, L: On the blow up phenomenon for the mass critical focusing Hartree equation in $\mathbb{R}^{4}$. Colloq. Math. 119, 23-50 (2010)

23. Ma, L, Zhao, L: Stability for the time-dependent Hartree equation with positive energy. J. Math. Anal. Appl. 362, 114-124 (2010)

24. Feng, B, Zhao, D: Optimal bilinear control of Gross-Pitaevskii equations with Coulombian potentials. J. Differ. Equ. 260 2973-2993 (2016)

\section{Submit your manuscript to a SpringerOpen ${ }^{\mathcal{O}}$ journal and benefit from:}

- Convenient online submission

- Rigorous peer review

- Open access: articles freely available online

- High visibility within the field

- Retaining the copyright to your article

Submit your next manuscript at $\mathbf{s p r i n g e r o p e n . c o m ~}$ 